\title{
Introducing a digital portfolio in pre-service teacher education Between aims and use: what is the coherence?
}

\author{
Walther Tessaro \\ University of Geneva
}

\begin{abstract}
Our paper aims to report on a study of how higher education students perceive and use an ePortfolio during their training. The analysis is based on a double theoretical corpus: research on the links between students' perceptions and learning approaches and those devoted to the use of the portfolio in higher education.

The perceptions collected from the students were compared with the reasons that led the trainers to integrate this tool in their training and assessment strategies. We examined the possible gap between perceptions and uses of the students and the educational intentions of their trainers. We also tried to put into perspective the use of the portfolio in relation to the context characteristics.

A triple data collection methodology was deployed including semi-structured interviews with tool designers and trainers, an online questionnaire survey for the students and semi-structured interviews with a sample of the same students.

The analyses highlight a discrepancy between the declared aims of the e-Portfolio tool and its uses. While both recognizing that the tool enabled their progress regarding expected skills and promoted the necessary reflectivity on their training and their future job, the students also noted a relative lack of usefulness of the portfolio in their professional development and knowledge integration.

It appears that the partial achievement of the ePortfolio's objectives can partly be explained through the poor and uneven support provided by trainers, whose mastering of this new educational and technological tool was missing as they had for most never used it nor were convinced by its value.
\end{abstract}

Keywords: ePortfolio, higher education, students' perception, assessment strategies 